\title{
Auditory Brainstem Responses to Bone-Conducted Brief Tones in Young Children with Conductive or Sensorineural Hearing Loss
}

\author{
Jennifer L. Hatton, ${ }^{1,2,3}$ Renée M. Janssen, ${ }^{1,3}$ and David R. Stapells ${ }^{1}$ \\ ${ }^{1}$ School of Audiology and Speech Sciences, The University of British Columbia, Vancouver, BC, Canada V6T 1Z3 \\ ${ }^{2}$ Audiology Department, Britsh Columbia's Children' Hospital, Vancouver, BC, Canada V6H 3V4 \\ ${ }^{3}$ British Columbia Early Hearing Program, Provicial Health Services Authority Victoria, BC, Canada V8V 3 K3
}

Correspondence should be addressed to David R. Stapells, stapells@audiospeech.ubc.ca

Received 4 April 2012; Accepted 29 June 2012

Academic Editor: Marcelo Rivolta

Copyright (C) 2012 Jennifer L. Hatton et al. This is an open access article distributed under the Creative Commons Attribution License, which permits unrestricted use, distribution, and reproduction in any medium, provided the original work is properly cited.

The bone-conduction (BC) tone ABR has been used clinically for over 20 years. The current study formally evaluated the test performance of the $\mathrm{BC}$ tone-evoked ABR in infants with hearing loss. Method. By comparing BC-ABR results to follow-up behavioural results, this study addressed two questions: (i) whether the BC tone ABR was successful in differentiating children with conductive versus sensorineural hearing loss (Study A; conductive: 68 ears; SNHL: 129 ears) and (ii) the relationship between BC ABR and behavioural hearing loss severity (Study B: $2000 \mathrm{~Hz}: 104$ ears; $500 \mathrm{~Hz}: 47$ ears). Results. Results demonstrate that the "normal" BC-ABR levels accurately differentiated normal versus elevated cochlear sensitivity (accuracy: $98 \%$ for $2000 \mathrm{~Hz}$; $98 \%$ for $500 \mathrm{~Hz}$ ). A subset of infants in Study A with elevated BC-ABR (i.e., no response at normal level) had additional testing at higher intensities, which allowed for categorization of the degree of cochlear impairment. Study B results indicate that the BC ABR accurately categorizes the degree of cochlear hearing loss for $2000 \mathrm{~Hz}$ (accuracy $=95.2 \%$ ). A preliminary dBnHL-to-dBHL correction factor of " $0 \mathrm{~dB}$ " was determined for $2000 \mathrm{~Hz}$ BC ABR. Conclusions. These findings further support the use of $\mathrm{BC}$ tone ABR for diagnostic ABR testing.

\section{Introduction}

Hearing loss is a relatively common sensory impairment that may have a wide variety of harmful effects on the affected child and family if not recognized and managed. Multiple studies have shown that children identified with hearing loss who have treatment and intervention services implemented no later than six months of age perform significantly better on various speech and language measures compared to children identified later (e.g., [1-3]). Thus, early hearing screening and intervention programs, such as the British Columbia Early Hearing Program (BCEHP), have the goal of screening all newborns for hearing loss by one month of age, comprehensive diagnostic assessment by three months of age, and implementation of intervention services and amplification no later than six months of age $[4,5]$.

The tone-evoked auditory brainstem response (ABR) is an important part of the diagnostic test battery and is currently the gold-standard technique for diagnosing type and degree of hearing loss in infants who do not pass hearing screening [5-7]. Several clinical ABR protocol guidelines have been established which recommend and describe the use of the tone-evoked ABR elicited using both airconducted (AC) and bone-conducted (BC) stimuli in order to characterize the degree and configuration of the hearing loss and to differentiate types (e.g., sensorineural or conductive) of hearing losses [4-6, 8]. Although tone-evoked ABR testing has been used successfully in both research and clinical settings since the 1980 s (for reviews, see [9-11]), surprisingly many clinicians today persist in using broadband click stimuli for ABR thresholds, even though the inadequacy of click-ABR threshold has been known and documented for many years (e.g., [12-16]). On a recent (March 22, 2012) search on the World Wide Web, many infant diagnostic ABR protocols were found that continue to use broadband click stimuli to determine ABR thresholds, and 
published surveys of clinicians' practices show they continue to use clicks (e.g., $[17,18])$.

A review of past and recent literature indicates that the tone-evoked ABR shows good correspondence with subsequent behavioural thresholds for AC stimuli. Correlations between $\mathrm{AC}$ tone $\mathrm{ABR}$ and behavioural thresholds are typically $r=.9$ or higher for $500 \mathrm{~Hz}, 2000 \mathrm{~Hz}$, and $4000 \mathrm{~Hz}$ (e.g., [19-21]). Across studies, most infants have toneevoked AC ABR thresholds within $5-10 \mathrm{~dB}$ of their puretone behavioural thresholds [10]. These results have been confirmed by others as well, and findings have been extended to include results from younger infants [20-25].

The most common cause of elevated ABR thresholds in young infants is conductive hearing loss $[7,26]$. This is especially so for young infants referred for diagnostic $A B R$ testing after failing one or more newborn hearing screenings. Diagnostic ABR protocols, therefore, must be able to determine whether a significant conductive component is present. When testing older children and adults behaviourally, this assessment is primarily achieved through comparison of airversus bone-conduction thresholds. For young infants, it is reasonable to expect that the finding of an elevated $A B R$ threshold to air-conduction stimuli with $A B R$ present to bone-conduction stimuli at normal levels would indicate the presence and degree of a conductive hearing loss; if ABR thresholds to bone-conduction stimuli are elevated, a sensorineural component is present $[15,16]$. As with airconduction testing, bone-conduction ABR testing must use frequency specific rather than broadband stimuli (i.e., brief tones rather than clicks) $[4-8,11]$. Current comprehensive diagnostic protocols for infants (e.g., $[4,8]$ ) emphasize the importance of obtaining bone-conduction information early in the $\mathrm{ABR}$ test sequence (i.e., as soon as an elevation in air-conduction thresholds is indicated) — this information is needed to determine the next test step and is important both for appropriate followup and for parent counselling. Unfortunately, many clinicians routinely fail to obtain ABR results for bone-conduction stimuli after finding elevated air-conduction threshold(s), relying instead on acoustic immittance results. However, a flat tympanogram cannot quantify the amount of conductive hearing loss and does not preclude SNHL; BC results are required.

Tone ABRs to bone-conducted stimuli also have a long history of research and clinical use, dating back at least 20 years [27-30]. The current Joint Committee on Infant Hearing $(\mathrm{JCIH})[5]$ guidelines emphasize the importance of $\mathrm{BC}-$ $\mathrm{ABR}$ testing for distinguishing between conductive and sensorineural hearing loss and tone-evoked BC-ABR testing is routinely used in large programs, such as the BCEHP [4] and the Ontario Infant Hearing Program (OIHP) [8]. Despite its clinical use and importance for clinical assessment, surprisingly few published data exist in the peer-reviewed literature regarding $\mathrm{BC}$ tone $\mathrm{ABR}$ testing in infants [20, 29-33]. Additionally, the majority of these published data pertain to infants having normal hearing or conductive hearing loss; very few data regarding $\mathrm{BC}-\mathrm{ABR}$ testing in infants with sensorineural hearing loss have been published.

The first study of the tone ABR to BC tones in infants was carried out by Stapells and Ruben [29], who investigated the tone ABR evoked by $500 \mathrm{~Hz}$ and $2000 \mathrm{~Hz}$ BC brief tones in 48 infants (mean age: 6.1 months) with either normal hearing (24 ears) or conductive hearing loss (41 ears). Their results demonstrated that the majority (94-100\%) of infants with normal cochlear function exhibit ABRs to BC stimuli at $30 \mathrm{dBnHL}$ for $2000 \mathrm{~Hz}$ and at $20 \mathrm{dBnHL}$ for $500 \mathrm{~Hz}$. These levels were suggested as the "normal" levels for clinical testing, separating infants with likely normal cochlear thresholds from those with likely sensorineural hearing loss $[15,16,34]$. These BC levels are currently used by many clinicians, including those involved with the BCEHP and the OIHP $[4,8]$. More recently, Vander Werff and colleagues [20] assessed BC-ABR thresholds in a group of infants with normal hearing $(500 \mathrm{~Hz}: N=40 ; 2000 \mathrm{~Hz}: N=40)$. Vander Werff and colleagues did not provide results which would allow determination of "normal" levels (i.e., cumulative response presence); however, mean $\mathrm{BC}$ tone $\mathrm{ABR}$ thresholds for the normal infants reported by Vander Werff and colleagues were similar (i.e., within $5-7 \mathrm{~dB}$, in $\mathrm{dB}$ re: $1 \mu \mathrm{N}$ ) to those reported for normal infants by Stapells and colleagues $[29,30]$. In addition to the above studies, two other studies have investigated $\mathrm{BC}$ tone- $\mathrm{ABR}$ thresholds in normal hearing infants. Foxe and Stapells [30] studied 500 and $2000 \mathrm{~Hz}$ BC-ABR responses in a small sample (8-9 infants; mean age 4.8 months) of normal hearing infants. Cone-Wesson and Ramirez [32] investigated BC-ABR thresholds at $500 \mathrm{~Hz}$ and $4000 \mathrm{~Hz}$, but not at $2000 \mathrm{~Hz}$, in a group of very young infants $(N=60$; aged 1-2 days $)$ at low risk for hearing loss. Notably, the $500 \mathrm{~Hz}$ BC-ABR thresholds of their very young infants were significantly lower (better) than those reported for older infants $[20,29,30]$, possibly related to maturation. It is difficult to compare these results due to the large age differences, as well as the lack of BC $2000 \mathrm{~Hz}$ data. A third study used a high-pass noise masking paradigm and demonstrated that infants' $\mathrm{BC}$ tone ABRs show reasonable cochlear place specificity, similar to that of adults [33]. Currently, there are too few infant ABR data for 1000 and $4000 \mathrm{~Hz} \mathrm{BC}$ tones to suggest appropriate normal levels for these frequencies; thus, current BCEHP and OIHP infant ABR protocols do not test these frequencies using boneconducted stimuli $[4,8,11]$.

Two studies have investigated the BC tone-ABR in groups of infants with conductive loss. Stapells and Ruben [29] recorded the $\mathrm{BC}$ tone-ABR in infants with otitis media $(25$ ears) or external auditory canal atresia (16 ears). Vander Werff and colleagues [20] looked at a group of infants with conductive hearing loss (500 Hz: 23 ears; $2000 \mathrm{~Hz}$ : 9 ears). Stapells and Ruben [29] demonstrated no differences in BCABR detectability at 500 and $2000 \mathrm{~Hz}$ in groups of infants with normal hearing and conductive hearing loss. Along the same line, Vander Werff and colleagues [20] showed that mean BC-ABR thresholds and latency-intensity functions were similar between infants with normal hearing and conductive hearing loss, suggestive of similar cochlear processing.

Very few published data exist regarding the $\mathrm{BC}$ tone ABR in infants with sensorineural hearing loss. Based on anecdotal case studies (e.g., $[10,11,27,35])$ and many years of clinical experience $[4,8,10]$, the $\mathrm{BC}$ tone $\mathrm{ABR}$ appears 
to accurately predict type of hearing loss. However, the only formal study is that of Vander Werff and colleagues [20], who obtained results from a very small group of infants with sensorineural hearing loss $(500 \mathrm{~Hz}: N=2 ; 2000 \mathrm{~Hz}: N=9)$. Furthermore, these researchers presented only ABR air-bone gap results and did not present any mean (or individual) BCABR thresholds. Results were not analyzed in such a way as to allow determination of $\mathrm{BC}-\mathrm{ABR}$ test performance.

As described above, the published $\mathrm{BC}$ tone-ABR literature, especially for infants with sensorineural hearing loss, is surprisingly lacking despite the many years of clinical experience. Importantly, to date, no study has evaluated the test performance of the tone-evoked $\mathrm{BC}-\mathrm{ABR}$ in infants. To address this, the current study compared $\mathrm{BC}$ tone-ABR results, obtained as part of a diagnostic ABR, with followup behavioural results for 108 infants with conductive or sensorineural hearing loss, with the goal of (i) investigating whether BC-ABR was successful in differentiating children with conductive versus sensorineural hearing loss (test performance) and (ii) determining the relationship between $\mathrm{BC}-\mathrm{ABR}$ and the subsequent severity of the behavioural cochlear hearing loss, if any.

\section{Methods}

2.1. Participants. Individuals with results included in this study were 108 young children referred to the British Columbia's Children's Hospital (BCCH) Audiology Department. The referrals for diagnostic $\mathrm{ABR}$ assessments were from both internal (i.e., within $\mathrm{BCCH}$ ) and external (community) sources. Children presented with a variety of risk factors (e.g., referral from hearing screening (note that $\mathrm{BCCH}$ is not one of the designated diagnostic sites for referrals from hearing screening in British Columbia. A small number of infants from screening are seen at $\mathrm{BCCH}$ in special circumstances, such as those who are very ill or who live in remote regions of the province), ototoxicity, delayed speech and language, neonatal insult, and/or other concomitant medical issues). The average age (corrected for prematurity) at the time of the initial diagnostic ABR assessment was 20.9 months $(\mathrm{SD}=16.6$; median $=18.7)$; the average amount of time between the ABR assessment and subsequent behavioural assessment was 13.3 months $(\mathrm{SD}=$ 14.9; median $=9.8$ ).

The chart review examined diagnostic ABR assessments that occurred between 2005 and 2011 and was approved by the Clinical Research Ethics Board of the University of British Columbia. The time period was chosen as it followed the introduction of a new tone-ABR protocol in the Audiology Department [4]. In general, assessments were included in the study if (i) BC-ABR testing had been completed at $2000 \mathrm{~Hz}$ and/or $500 \mathrm{~Hz}$, and (ii) reliable behavioural results had been obtained for the child subsequent to the ABR assessment. As a result of requiring $\mathrm{BC}-\mathrm{ABR}$ testing for inclusion, all $\mathrm{ABR}$ assessments included in this study involved abnormal AC ABR thresholds (hearing thresholds $\geq 30 \mathrm{~dB}$ estimated behavioural hearing level (eHL)) for at least one of 500 and $2000 \mathrm{~Hz}$, as these are the two frequencies at which BC-ABR recordings are obtained (as per the BCCH/BCEHP ABR protocol, BC recordings are presently only obtained for 500 and $2000 \mathrm{~Hz}$ and only if AC thresholds are elevated. $\mathrm{BC}$ recordings for other frequencies are not obtained as normative data and criterion values for "normal" levels have not been established [4]). If multiple behavioural test results were obtained for a given subject, the behavioural test closest in time to ABR testing and/or with most complete results and good reliability was chosen.

The chart review indicated that 558 children had undergone diagnostic ABR assessments during the period of this review. Approximately half $(46 \%)$ of the children in the initial chart review were excluded because they had normal ABR results and therefore no $\mathrm{BC}-\mathrm{ABR}$ results. Additional reasons for exclusion were (i) no reliable behavioural results were available, (ii) the ABR indicated a unilateral loss (i.e., normal hearing for one ear) and subsequent behavioural testing was only obtained in soundfield, (iii) the hearing loss was clearly progressive, (iv) the child had evidence of auditory neuropathy spectrum disorder, or (v) BC-ABR results were inconclusive. After exclusion, results for 108 infants were included. Not all infants had hearing loss in both ears and/or had complete results (i.e., both ears with both 500 and $2000 \mathrm{~Hz}$ results); therefore, the study includes results for a maximum of 138 ears at $2000 \mathrm{~Hz}$ and 59 ears at $500 \mathrm{~Hz}$.

2.2. Sedation. Generally, patients seen for ABR assessments aged over six months were sedated to ensure sufficient sleep time (87/108 participants). The sedative, when utilized, was chloral hydrate, prescribed by the patients' otolaryngologist, and administered and monitored by the sedation clinic nurse, similar to the procedure described in the American Academy of Pediatrics Guidelines [36, 37].

2.3. ABR Parameters. All assessments were carried out in a double-walled, sound-attenuated booth using the Intelligent Hearing Systems (IHS) SmartEP system. The ABR was assessed to air conduction at 500,1000, 2000, and $4000 \mathrm{~Hz}$; bone conduction at 500 and $2000 \mathrm{~Hz}$, where indicated, and to high-intensity clicks, where indicated. Details of the $\mathrm{BCCH} / \mathrm{BCEHP} \mathrm{ABR}$ protocols and parameters are available on the web [4] and in the literature [11]. Briefly, AC-ABR stimuli were presented using insert earphones (Etymotic ER$3 \mathrm{~A}$ ) and BC-ABR stimuli were presented via a Radioear B71 bone vibrator. Stimuli were brief tones (5-cycle duration, exact-Blackman window) presented at a rate of 39.1/second. $\mathrm{AC}$ and $\mathrm{BC}$ stimuli were calibrated in $\mathrm{dBnHL}$ using $0 \mathrm{dBnHL}$ calibrations provided by Stapells [11] (normal hearing levels (nHL) for AC- and BC-ABR stimuli are referenced to adult behavioural thresholds, as are hearing threshold levels (HL) for paediatric behavioural testing). ABRs were recorded with the noninverting electrode placed on the high forehead (Fpz) and inverting electrodes on the ipsilateral and, for BC ABR, contralateral mastoids. An electrode placed on the forehead served as ground. This setup allowed the simultaneous recording of two EEG channels ((i) high forehead to left mastoid (Fpz-M2), and (ii) high forehead to right mastoid (Fpz-M1)) which were obtained for all BC-ABR recordings 
(as per the BCCH/BCEHP ABR protocol, comparison of the ipsilateral (i.e., side where stimulus transducer is placed) and contralateral EEG channel ABR recordings is used to determine responding ear. Normally, a larger or earlier wave $\mathrm{V}$ in the ipsilateral EEG channel indicates the responding cochlea; however, a larger or earlier response in the contralateral EEG channel is abnormal and suggests the contralateral ear is producing the response $[11,29]$. In the current study, for 101 infants, results in the contralateral EEG channel did not change $\mathrm{BC}-\mathrm{ABR}$ interpretation (i.e., responses were either absent in both channels or the ipsilateral response was clearly better). In only seven infants was the interpretation changed by a larger or earlier response in the contralateral channel). Interelectrode impedances were less than $3 \mathrm{kOhms}$ at $30 \mathrm{~Hz}$. The EEG was amplified and filtered using a bandpass of 30$1500 \mathrm{~Hz}$. Trials containing amplitudes greater than $\pm 25 \mu \mathrm{V}$ were automatically rejected. Averaging was carried out using a $24.6 \mathrm{~ms}$ poststimulus analysis time. At least two to three replications of 2000 trials each was always obtained for threshold bracketing conditions.

2.4. Diagnostic ABR Protocol. The BCCH/BCEHP tone-ABR protocol test sequence emphasizes efficiency and obtaining information in a prioritized fashion. A detailed description of this diagnostic ABR protocol is freely available on the World Wide Web [4]. Key features of the protocol are (1) to commence testing at a low (target level for "normal") intensity ("Normal" dBnHL values are as follows: AC $500 \leq$ 35 , AC $1000 \leq 35$, AC $2000 \leq 30$, AC $4000 \leq 25$, BC $500 \leq 20$, and BC $2000 \leq 30$ ); (2) to switch ears immediately and frequently; (3) to obtain recordings to $\mathrm{BC}$ stimuli as soon as it has been determined that an AC response is elevated; (4) to initially use a relatively large step size (i.e., greater than $10 \mathrm{~dB}$ ) that allows for a quick bracketing of thresholds, when elevated, and end with a maximum step size of $10 \mathrm{~dB}$. If a clear reproducible response is obtained to AC recordings at the target "normal" intensity, that ear/frequency is said to be "normal," and threshold below this normal intensity is not obtained. If AC responses are not present at the normal intensity (i.e., elevated AC), BC recordings are obtained to determine whether an elevated $\mathrm{AC}$ threshold is conductive or sensorineural in nature; however, it is not used to estimate the size of the air-bone gap, if present. Until recently [4], actual BC-ABR thresholds were not routinely pursued; rather, testing was often only done at the normal BC intensity and, if no response was present, then at the maximum BC intensity (60 dBnHL for $2000 \mathrm{~Hz} ; 50 \mathrm{dBnHL}$ for $500 \mathrm{~Hz}$ ). Testing is initiated at AC $2000 \mathrm{~Hz}$ (both ears sequentially), and proceeds directly to $\mathrm{BC} 2000 \mathrm{~Hz}$ for those ear(s) for which AC $2000 \mathrm{~Hz}$ is elevated. $500 \mathrm{~Hz}$ (AC and BC) is given second priority after $2000 \mathrm{~Hz}$.

\subsection{Classification}

2.5.1. ABR-Based Sensorineural versus Conductive Hearing Loss Categorization. ABR-based categorization was carried out and analyzed for each frequency $(2000$ and $500 \mathrm{~Hz})$ independently. ABR results were included in the sensorineural group for each frequency if $\mathrm{AC}$ - and $\mathrm{BC}-\mathrm{ABR}$ results were elevated at that frequency. ABR results were included in the conductive group for each frequency if $\mathrm{AC} A \mathrm{ABR}$ results were elevated and $\mathrm{BC}-\mathrm{ABR}$ results were normal at that frequency.

2.5.2. Behavioural Follow-Up Classification Scheme. BC-ABR results were compared to behavioural results. However, not all children had BC testing completed at the time of behavioural followup; thus, behavioural results were divided into two subgroups, depending on whether or not BC behavioural results were available for those participants with AC elevations. Participants with "certain" hearing status had either normal AC behavioural thresholds on followup (e.g., resolution of a transient conductive hearing loss) or had followup BC behavioural results available $(2000 \mathrm{~Hz}$ : 82 ears; $500 \mathrm{~Hz}$ : 50 ears). However, not all the participants satisfied the criteria for "certain" hearing status. For these additional children, the combination of elevated AC behavioural thresholds and normal acoustic immittance results allowed us to infer elevated cochlear hearing status. These children without follow-up BC behavioural results were included in the "presumed SNHL" group $(2000 \mathrm{~Hz}$ : 56 ears; $500 \mathrm{~Hz}$ : 9 ears), thereby increasing the overall sample size of the SNHL group (data for ears with abnormal middle-ear status and no $\mathrm{BC}$ results at the time of behavioural assessment were excluded because type of hearing loss could not be determined).

2.6. Study A: Test Performance: Accuracy of BC-ABR in Evaluating Type of Hearing Loss (SNHL versus $C H L$ ). This study first evaluated the accuracy of $\mathrm{ABR} \mathrm{BC}$ recordings in correctly characterizing type of hearing loss in 108 infants with hearing loss. Participants were categorized as having hearing loss of either sensorineural $(N=129$ ears $)$ or conductive ( $N=68$ ears) type, based on the results of the ABR assessment. The number of infants who were correctly identified as per subsequent behavioural testing as having either CHL or SNHL was then determined.

\subsection{Study B: BC-ABR Severity and Degree of Hearing Loss:} Relationship between $B C-A B R$ Results and $A C$ Behavioural Thresholds. Study B evaluated the relationship between BC$\mathrm{ABR}$ and $\mathrm{AC}$ behavioural results. At the time of this study, the $\mathrm{BCEHP}$ protocol did not mandate that BC-ABR thresholds be obtained; therefore, only a subset of the infants included in the first part of this study had BC-ABR results completed at multiple intensities. Results of a total of 85 infants were included in Study B. Seventy infants (104 ears) had $2000 \mathrm{~Hz}$ BC-ABR thresholds that fell within the following three categories: (i) $\leq 30 \mathrm{dBnHL}$ (i.e., normal; 29 ears); (ii) 35-60 dBnHL (elevated; 26 ears); (iii) $>60 \mathrm{dBnHL}$ (no response at maximum intensity; 49 ears). Twenty-one ears had actual BC-ABR thresholds obtained at $2000 \mathrm{~Hz}$. Thirtyeight infants (47 ears) had $500 \mathrm{~Hz}$ BC-ABR thresholds that fell within the following three categories: (i) $\leq 20 \mathrm{dBnHL}$ (i.e., normal; 37 ears), (ii) 25-50 dBnHL (i.e., elevated; 6 ears), and (iii) > $50 \mathrm{dBnHL}$ (no response at maximum intensity; 4 ears). Six ears had actual BC-ABR thresholds obtained 


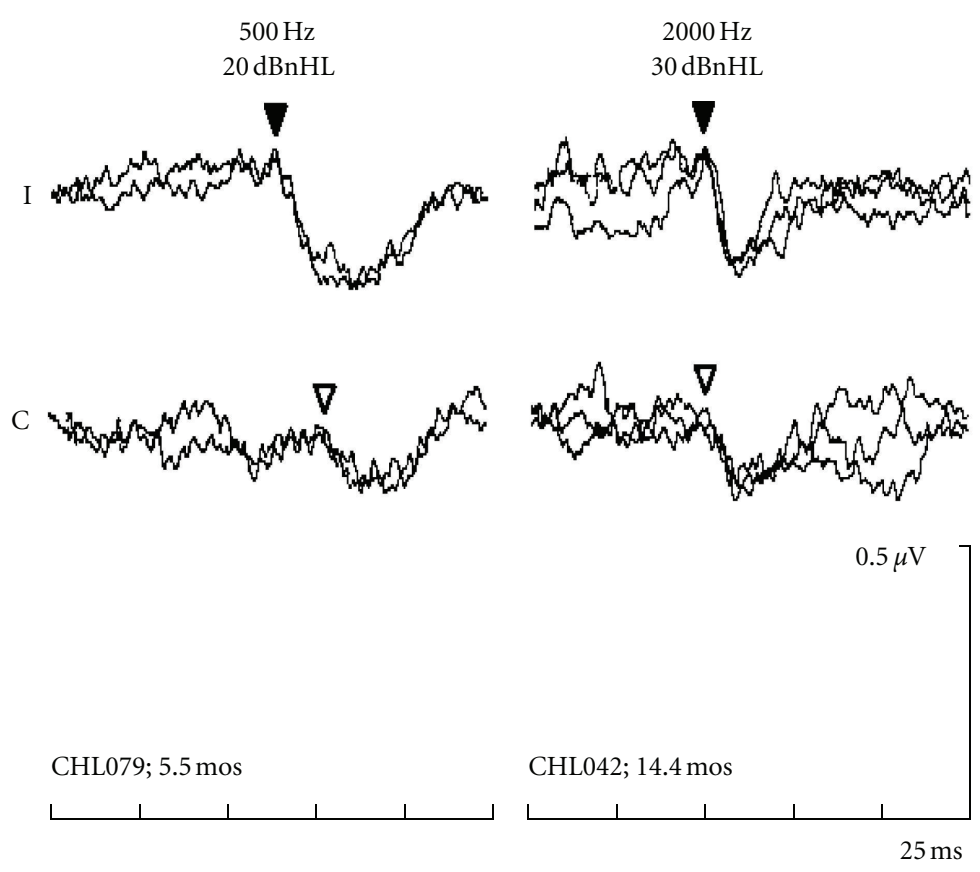

FIGURE 1: ABRs to 500 and $2000 \mathrm{~Hz}$ BC tones, presented at 20 and $30 \mathrm{dBnHL}$ respectively, recorded from two infants with conductive hearing loss at the time of ABR testing. Age at ABR testing indicated on the bottom. These infants had normal hearing to air-conduction stimuli at behavioural followup. "I" equals the ipsilateral channel (EEG channel ipsilateral to the BC transducer). "C" equals the contralateral channel (EEG channel contralateral to the BC transducer). Waves V are indicated by the triangles (filled $=$ ipsilateral; open $=$ contralateral).

at $500 \mathrm{~Hz}$. Study B compared the correspondence between these three BC-ABR categories with behavioural outcomes. For the small number of infants for which actual BC-ABR thresholds were obtained, threshold difference scores (i.e., $\mathrm{BC}-\mathrm{ABR}$ minus AC or BC behavioural) were calculated.

\section{Results}

3.1. Study A. This study evaluated the accuracy of BC$\mathrm{ABR}$ recordings to correctly identify conductive versus sensorineural hearing loss. Figure 1 shows 500 and $2000 \mathrm{~Hz} \mathrm{BC-}$ $\mathrm{ABR}$ results present at the normal BCEHP levels recorded from two infants-one infant at $500 \mathrm{~Hz}$ (left); the other infant at $2000 \mathrm{~Hz}$ (right)—both with elevated AC ABR results (i.e., conductive hearing loss). Typical for infants with normal cochlear sensitivity, these infants' waves $\mathrm{V}$ in the ipsilateral EEG channels are earlier and larger than those seen in the contralateral EEG channels $[16,29]$. Behavioural testing was consistent with normal hearing.

Figure 2 demonstrates "elevated" BC-ABR results (i.e., no response at the normal intensities) to 500 and $2000 \mathrm{~Hz}$ bone-conduction tones recorded from two infants with elevated $\mathrm{AC}$ - and $\mathrm{BC}-\mathrm{ABR}$ results (i.e., sensorineural hearing loss). No clear wave $\mathrm{V}$ is detected at the normal BC-ABR levels. For the infant with $500 \mathrm{~Hz}$ results, behavioural testing at age 24 months indicated a profound sensorineural hearing loss. For the infant with $2000 \mathrm{~Hz}$ results, behavioural testing at 41 months was consistent with moderate sensorineural hearing loss.
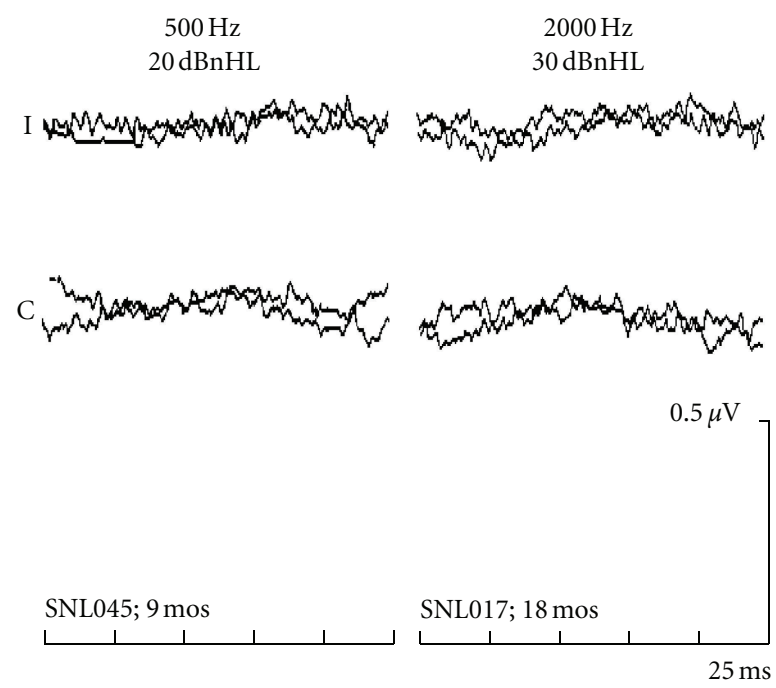

FIGURE 2: "Elevated" ABR results (i.e., no response at normal intensities) for $500-$ and $2000-\mathrm{Hz} \mathrm{BC}$ tones recorded from two infants with sensorineural hearing loss. Age at ABR testing indicated on the bottom. On behavioural follow up, both infants had sensorineural hearing loss.

The examples in Figures 1 and 2 are typical of the results obtained from the group of infants in Study A. Table $1 \mathrm{com}-$ pares BC-ABR determination of cochlear status with actual cochlear status as determined by behavioural followup. The $\mathrm{BC}-\mathrm{ABR}$ results accurately categorized cochlear status, erring 
TABLE 1: Accuracy of "normal" BC-ABR levels for classification of "normal" versus "elevated" cochlear status.

\begin{tabular}{lcccc}
\hline & & & \multicolumn{2}{c}{ BC-ABR results } \\
& & & Normal & Elevated \\
\hline $2000 \mathrm{~Hz}$ & Behavioural outcome & Normal & $28(20.3 \%)$ & $0(0.0 \%)$ \\
138 ears & & Elevated & $3(2.2 \%)$ & $107(77.5 \%)$ \\
\hline $500 \mathrm{~Hz}$ & Behavioural outcome & Normal & $36(61.0 \%)$ & $0(0.0 \%)$ \\
59 ears & & Elevated & $1(1.7 \%)$ & $22(37.3 \%)$ \\
\hline
\end{tabular}

Percent of total ears in parentheses.

BC ABR "normal" levels: 30 and $20 \mathrm{dBnHL}$ for 2000 and $500 \mathrm{~Hz}$.

Data for the "certain" and "presumed SNHL" groups included.

in only $2.0 \%(4 / 197)$ of the comparisons (across frequencies), with similar results overall for the two frequencies. Results were equally accurate when only the "certain" data were considered. Of the four errors, three had mild sensorineural hearing loss (30-40 dBHL) and one had moderate sensorineural hearing loss on followup that was missed by BC ABR. Overall, there were 13 comparisons with mild sensorineural hearing loss in Study A; the ABR missed only three of these mild losses.

Results of Study A demonstrate, for a large number of infants, that the BC-ABR normal levels of $30 \mathrm{dBnHL}$ for $2000 \mathrm{~Hz}$ and $20 \mathrm{dBnHL}$ for $500 \mathrm{~Hz}$ accurately differentiate normal versus elevated cochlear sensitivity in infants with conductive or sensorineural hearing loss. Across both frequencies, sensitivity was $97 \%$; specificity was $100 \%$. These results are consistent with clinical experience and corroborate clinical ABR protocols used by the BCEHP [4] and other programs (e.g., $[8,16])$.

3.2. Study B. Although the results of Study A showed that $\mathrm{BC}-\mathrm{ABR}$ is accurate in identifying the type of hearing loss, because these BC data were only obtained at the "normal" levels, Study A was not able to provide information regarding the degree of cochlear impairment. However, a subset of infants with elevated BC-ABR results in Study A were also tested at higher $\mathrm{BC}$ intensities, usually at the maximum allowable BC-ABR intensities (i.e., $60 \mathrm{dBnHL}$ for $2000 \mathrm{~Hz}$ and $50 \mathrm{dBnHL}$ for $500 \mathrm{~Hz}$ ). By comparing these results to behavioural followup, this allowed us to assess how well BCABR categorized the severity of cochlear impairment.

Figure 3 presents results for $2000 \mathrm{~Hz}$ from three young children, typical of the group results, where $\mathrm{BC}-\mathrm{ABR}$ was used to categorize cochlear sensitivity into three categories: (i) normal, (ii) 35-60 dBnHL, and (iii) $>60 \mathrm{dBnHL}$. Results for the 3.4-month-old infant on the left panel indicate normal cochlear sensitivity, which was confirmed at behavioural followup at 32 months of age. The middle panel shows results for a 5-year-old indicating elevated, but present at $40 \mathrm{dBnHL}, \mathrm{BC}-\mathrm{ABR}$ results; behavioural followup was consistent with a moderate SNHL. The right panel displays BC-ABR results from an infant with profound SNHL (determined by behavioural followup) showing absent BC$\mathrm{ABR}$ at the maximum level of $60 \mathrm{dBnHL}$.

Figure 4 displays typical results for $500 \mathrm{~Hz}$ from three young children. Similar to the $2000 \mathrm{~Hz}$ results described above, BC-ABR was used to categorize cochlear sensitivity into three categories: (i) normal, (ii) $25-45 \mathrm{dBnHL}$, and (iii) $>50 \mathrm{dBnHL}$. The left panel shows results from a 3.4-monthold infant with normal cochlear sensitivity; behavioural followup revealed normal hearing. The middle panel shows results for a 21-month-old infant indicating elevated BC$\mathrm{ABR}$ results, but a clear response present at $40 \mathrm{dBnHL}$; behavioural followup was consistent with a moderate SNHL. Results for the 14-month-old infant with profound SNHL on the right panel show absent $\mathrm{BC}-\mathrm{ABR}$ at the maximum level of $50 \mathrm{dBnHL}$.

The above examples in Figures 3 and 4 indicate that the BC-ABR was able to appropriately categorize degree of cochlear impairment as determined by behavioural testing. In order to assess results for the group data, two comparisons were made between $\mathrm{BC}-\mathrm{ABR}$ and behavioural outcome results. First, we compared BC-ABR to behavioural AC thresholds to maximize the number of data points (there were many instances where behavioural $\mathrm{BC}$ thresholds were not available); AC behavioural results were only used when there was evidence of no conductive overlay. In cases of permanent conductive hearing loss, where behavioural AC thresholds were not reflective of cochlear sensitivity, behavioural BC results were used in place of AC thresholds. In a subsequent analysis, we focused specifically on comparisons between $\mathrm{BC}-\mathrm{ABR}$ and $\mathrm{BC}$ behavioural, which resulted in a smaller sample size for these latter analyses.

Figure 5 shows the results of the first comparison of BCABR category to (primarily) AC behavioural threshold data for $2000 \mathrm{~Hz}$ (Figure 5(a); 104 ears) and $500 \mathrm{~Hz}$ (Figure 5(b); 47 ears). With only a few exceptions, as BC-ABR category increases in severity so do AC behavioural thresholds. Although present for both frequencies, there are fewer data for $500 \mathrm{~Hz}$ in the more severe hearing loss categories, and therefore conclusions are limited for this frequency.

Based on the results of Figure 5, we divided the behavioural results into three categories of severity of hearing impairment and then quantified how well the BC-ABR categories corresponded to these three behavioural threshold categories. Table 2 shows the results of these comparisons. For $2000 \mathrm{~Hz}$, where the most data are available, the Spearman rank correlation coefficient was .96; BC-ABR correctly determined behavioural hearing loss category in $95.2 \%$ of the comparisons. For $500 \mathrm{~Hz}$, the Spearman rank correlation coefficient was .95; BC-ABR correctly determined behavioural hearing loss category in $95.7 \%$ of the comparisons. As mentioned previously, the data for $500 \mathrm{~Hz}$ are limited due 
TABLE 2: Comparison of BC-ABR category to AC behavioural threshold data for $2000 \mathrm{~Hz}$ and $500 \mathrm{~Hz}$.

\begin{tabular}{|c|c|c|c|c|c|}
\hline & & & \multicolumn{3}{|c|}{ BC-ABR category (dBnHL) } \\
\hline \multirow{4}{*}{$\begin{array}{l}2000 \mathrm{~Hz} \\
104 \text { ears }\end{array}$} & \multirow{4}{*}{$\begin{array}{l}\text { Behavioural outcome } \\
\text { category }(\mathrm{dBHL})\end{array}$} & & Normal & $35-60$ & $>60$ \\
\hline & & Normal & $28(26.9 \%)$ & $0(0 \%)$ & $0(0 \%)$ \\
\hline & & $30-65$ & $1(1.0 \%)$ & $25(24.0 \%)$ & $3(2.9 \%)$ \\
\hline & & $>65$ & $0(0 \%)$ & $1(1.0 \%)$ & $46(44.2 \%)$ \\
\hline \multirow{4}{*}{$\begin{array}{l}500 \mathrm{~Hz} \\
47 \text { ears }\end{array}$} & \multirow{4}{*}{$\begin{array}{l}\text { Behavioural outcome } \\
\text { category }(\mathrm{dBHL})\end{array}$} & & Normal & $25-45$ & $>50$ \\
\hline & & Normal & $36(76.6 \%)$ & $0(0 \%)$ & $0(0 \%)$ \\
\hline & & $30-55$ & $1(2.1 \%)$ & $5(10.6 \%)$ & $0(0 \%)$ \\
\hline & & $>55$ & $0(0 \%)$ & $1(2.1 \%)$ & $4(8.5 \%)$ \\
\hline
\end{tabular}

Percent of total ears in parentheses.

BC ABR "normal" levels: 30 and $20 \mathrm{dBnHL}$ for 2000 and $500 \mathrm{~Hz}$.

$\mathrm{AC}$ (and BC) behavioural "normal" levels are $\leq 25 \mathrm{dBHL}$.

Most behavioural threshold data are AC (2000 Hz: 89/104; $500 \mathrm{~Hz}: 33 / 47)$. See text for details.

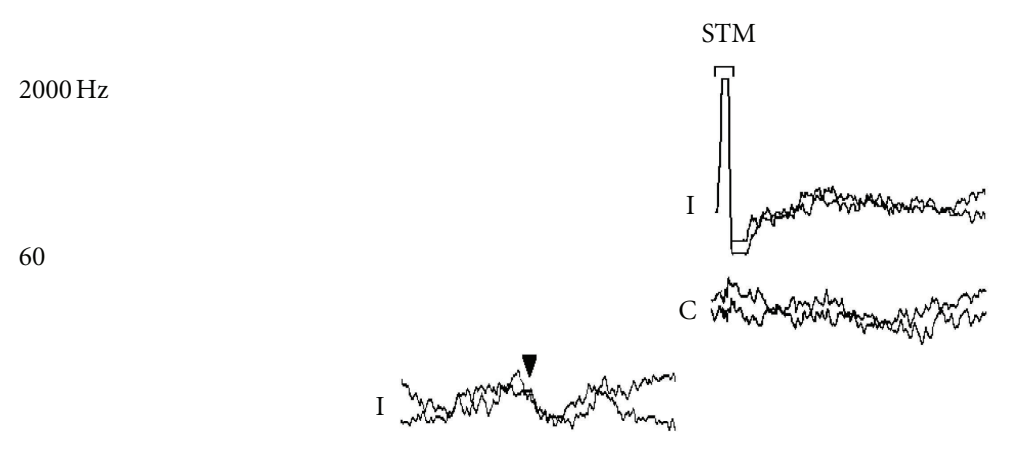

40
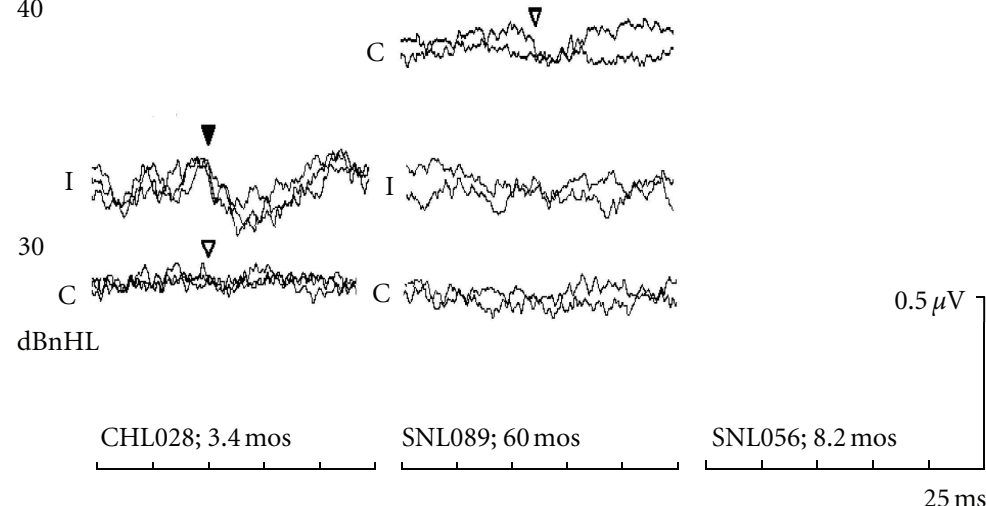

FIGURE 3: Left panel: ABRs to $30 \mathrm{dBnHL}$ BC $2000 \mathrm{~Hz}$ tones recorded from an infant with conductive hearing loss at the time of ABR testing, who had normal hearing at behavioural followup. Middle Panel: "Elevated" $2000 \mathrm{~Hz}$ BC-ABR results (threshold $=40 \mathrm{dBnHL}$ ) from a child who showed a moderate sensorineural hearing loss at behavioural followup. Right panel: "Elevated" $2000 \mathrm{~Hz}$ BC-ABR results (threshold > $60 \mathrm{dBnHL}$ ) from an infant who showed profound sensorineural hearing loss behaviourally. STM indicates the region of uncancelled stimulus artifact.

to the small number of participants with hearing loss in this analysis with BC-ABR results above the normal level.

Figure 6 shows the results of the second comparison where we compared the BC-ABR only to behavioural BC threshold data for $2000 \mathrm{~Hz}$ (Figure 6(a); 41 ears) and $500 \mathrm{~Hz}$ (Figure 6(b); 19 ears). Because of the more restrictive inclusion criteria, sample's sizes are smaller, especially at $500 \mathrm{~Hz}$. Similar to the results shown in Figure 5, $2000 \mathrm{~Hz}$ results show that as $\mathrm{BC}-\mathrm{ABR}$ hearing loss severity category increases so does $\mathrm{BC}$ behavioural threshold. We do not see the large discrepancies between $\mathrm{BC}-\mathrm{ABR}$ and $\mathrm{BC}$ behavioural as we did when comparing $\mathrm{BC}-\mathrm{ABR}$ to $\mathrm{AC}$ behavioural. For $500 \mathrm{~Hz}$, there is a suggestion of a correspondence between $\mathrm{BC}-\mathrm{ABR}$ category and $\mathrm{BC}$ behavioural thresholds, but due to limited data with hearing loss, results are inconclusive.

Table 3 shows the results of the comparisons in Figure 6. For $2000 \mathrm{~Hz}$, the Spearman rank correlation coefficient was .96; BC-ABR correctly determined behavioural hearing loss category in $92.8 \%$ of the comparisons. For $500 \mathrm{~Hz}$, the Spearman rank correlation coefficient was .93; BC-ABR correctly 
$500 \mathrm{~Hz}$

50

I

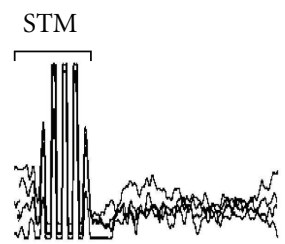

40
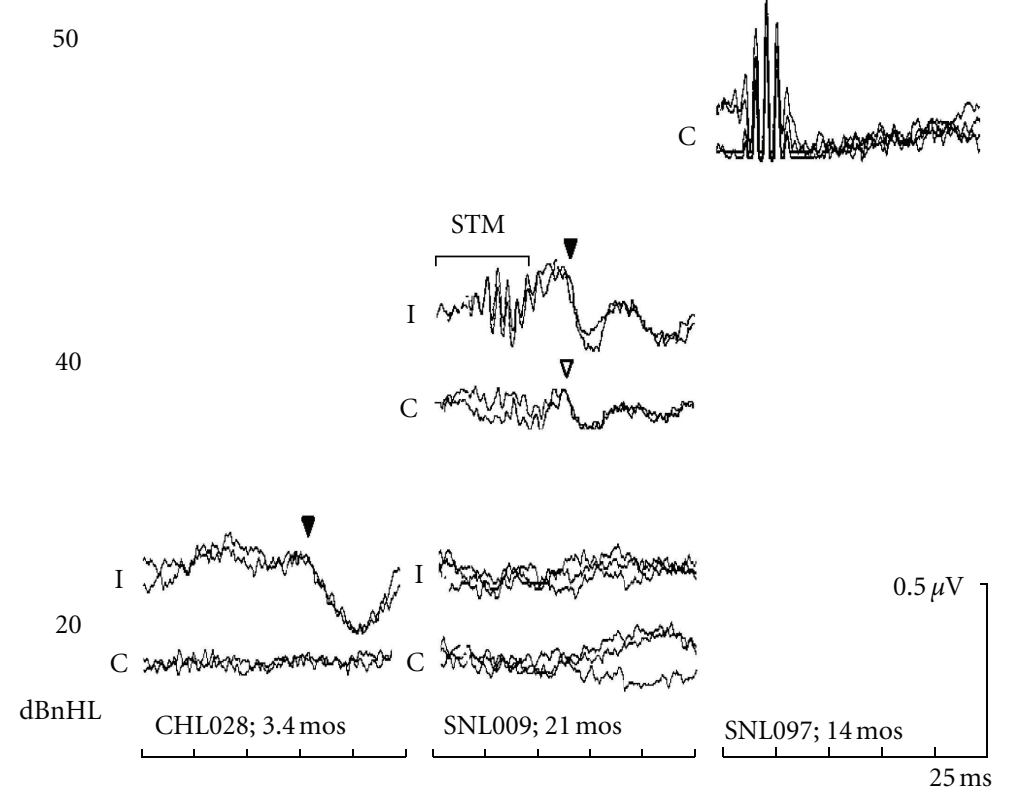

Figure 4: Left panel: ABRs to $20 \mathrm{dBnHL}$ BC $500 \mathrm{~Hz}$ tones recorded from an infant with conductive hearing loss at the time of ABR testing, who showed normal hearing at behavioural followup. Middle panel: "Elevated" $500 \mathrm{~Hz}$ BC-ABR results (threshold $=30-40 \mathrm{dBnHL}$ ) from an infant who showed a moderate sensorineural hearing loss behaviourally. Right panel: "Elevated" $500 \mathrm{~Hz}$ BC-ABR results (threshold > $50 \mathrm{dBnHL}$ ) from an infant with a severe/profound sensorineural hearing loss bilaterally at followup. STM indicates the region of uncancelled stimulus artifact.

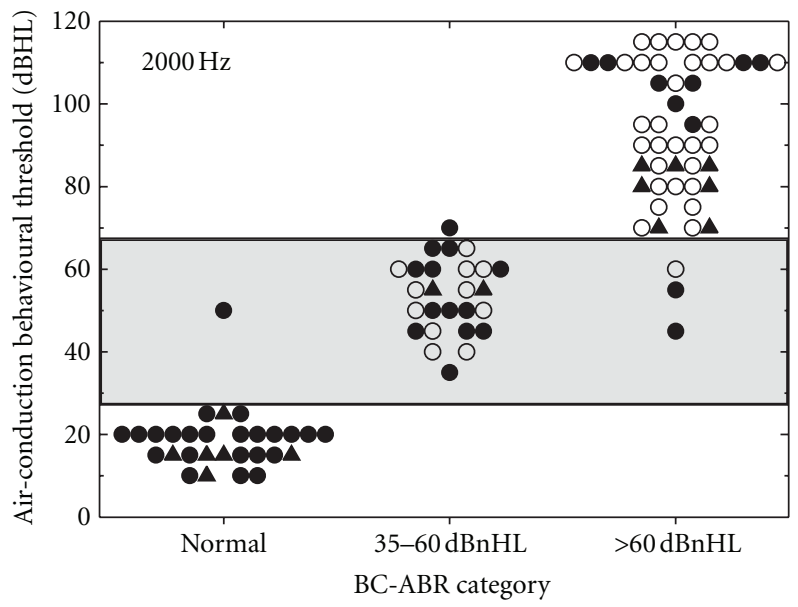

(a)

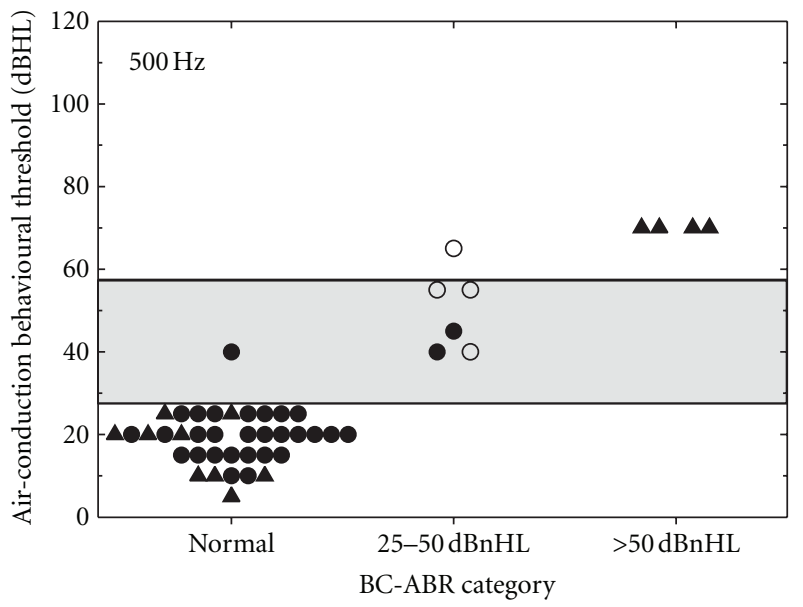

(b)

FIGURE 5: Comparison of BC-ABR threshold category to AC behavioural threshold data for $2000 \mathrm{~Hz}$ (a) and $500 \mathrm{~Hz}$ (b). Filled circles indicate ears with "certain" hearing status, whereas open circles indicate ears with "presumed SNHL." Filled triangles represent ears where $\mathrm{BC}$ behavioural results were used (i.e., when AC behavioural results did not reflect cochlear status). Behavioural categories are indicated by horizontal lines/shading. See text for details. 
TABLE 3: Comparison of BC-ABR category to BC behavioural threshold data for $2000 \mathrm{~Hz}$ and $500 \mathrm{~Hz}$.

\begin{tabular}{|c|c|c|c|c|c|}
\hline & & & \multicolumn{3}{|c|}{ BC-ABR category (dBnHL) } \\
\hline \multirow{4}{*}{$\begin{array}{l}2000 \mathrm{~Hz} \\
41 \text { ears }\end{array}$} & \multirow{4}{*}{$\begin{array}{l}\text { Behavioural outcome } \\
\text { category }(\mathrm{dBHL})\end{array}$} & & Normal & $35-60$ & $>60$ \\
\hline & & Normal & $9(22.0 \%)$ & $0(0 \%)$ & $0(0 \%)$ \\
\hline & & $30-65$ & $1(2.4 \%)$ & $15(36.6 \%)$ & $1(2.4 \%)$ \\
\hline & & $>65$ & $0(0 \%)$ & $0(0 \%)$ & $15(36.6 \%)$ \\
\hline \multirow{4}{*}{$\begin{array}{l}500 \mathrm{~Hz} \\
19 \text { ears }\end{array}$} & \multirow{4}{*}{$\begin{array}{l}\text { Behavioural outcome } \\
\text { category }(\mathrm{dBHL})\end{array}$} & & Normal & $25-45$ & $>50$ \\
\hline & & Normal & $13(68.4 \%)$ & $0(0 \%)$ & $0(0 \%)$ \\
\hline & & $30-55$ & $1(5.3 \%)$ & $1(5.3 \%)$ & $0(0 \%)$ \\
\hline & & $>55$ & $0(0 \%)$ & $0(0 \%)$ & $4(21.1 \%)$ \\
\hline
\end{tabular}

Percent of total ears in parentheses.

BC ABR "normal" levels: 30 and $20 \mathrm{dBnHL}$ for 2000 and $500 \mathrm{~Hz}$.

BC behavioural "normal" levels are $\leq 25$ dBHL.

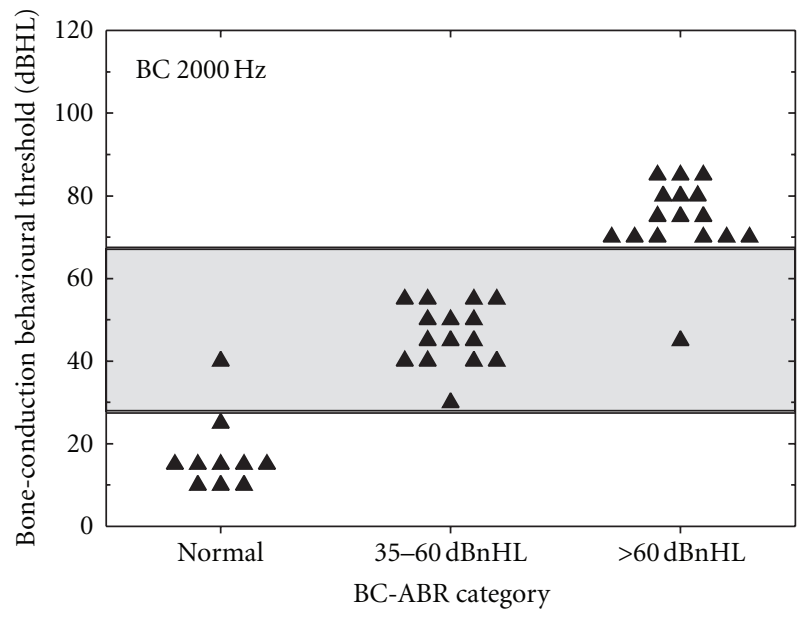

(a)

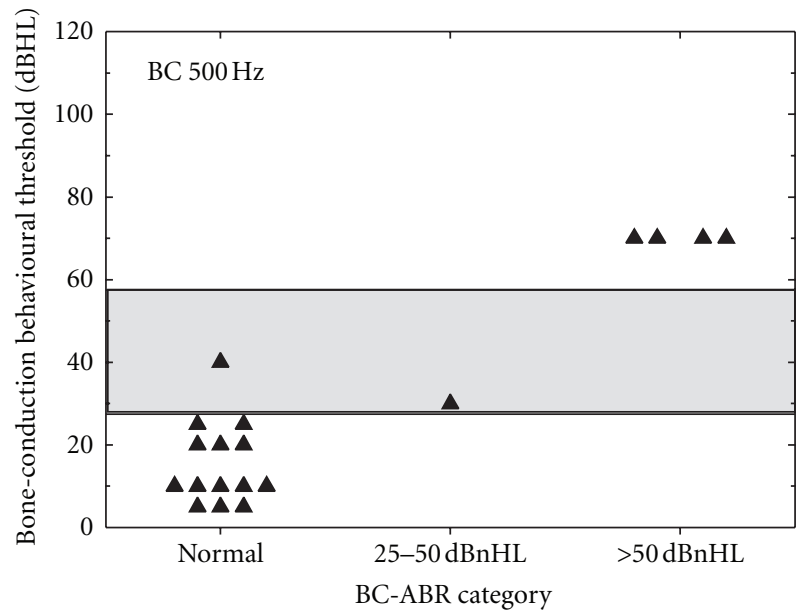

(b)

Figure 6: Comparison of BC-ABR threshold category to BC behavioural threshold data for $2000 \mathrm{~Hz}$ (a) and $500 \mathrm{~Hz}$ (b). Behavioural categories are indicated by horizontal lines/shading.

determined behavioural hearing loss category in $94.7 \%$ of the comparisons. As noted above, due to limited data for hearing loss, the results of this analysis are inconclusive for $500 \mathrm{~Hz}$.

The preceding analysis was unable to provide ABRminus-behavioural threshold difference scores for the purpose of determining correction factors because they included nonthreshold data (e.g., response present at the normal levels indicating normal cochlear sensitivity; no response at the maximum intensity levels). Nevertheless, there was a subset of cases in the middle BC-ABR category where actual BC$\mathrm{ABR}$ thresholds were obtained and thus difference scores could be calculated. For $2000 \mathrm{~Hz}, 21$ ears had both BC-ABR and $\mathrm{AC}$ behavioural thresholds, with the $\mathrm{BC}-\mathrm{ABR}$ on average better than behavioural thresholds (mean $=-6.2 \mathrm{~dB}$; $\mathrm{SD}=$ $8.9 ; t=-3.2, d f=20, P<.005)$. Most $(71 \%)$ of these comparisons were within $10 \mathrm{~dB}, 91 \%$ were within $15 \mathrm{~dB}$, and all were within $25 \mathrm{~dB}$. Only 2 ears $(9.5 \%)$ showed BC-ABR thresholds worse than AC behavioural thresholds.

Twelve ears had both ABR and behavioural BC thresholds at $2000 \mathrm{~Hz}$, with the BC ABR on average being slightly but not significantly better $($ mean $=-1.3 \mathrm{~dB} ; \mathrm{SD}=8.3$; $t=-0.5, d f=11, P=.61)$. Most $(83.3 \%)$ of these comparisons were within $5 \mathrm{~dB}, 92 \%$ were within $15 \mathrm{~dB}$, and all were within $20 \mathrm{~dB}$. Again, BC ABR was better than or equal to behavioural $\mathrm{BC}$ thresholds in most cases. Only 2 ears $(17 \%)$ showed BC-ABR thresholds worse than BC behavioural thresholds.

Threshold difference scores for $500 \mathrm{~Hz}$ were available for only 6 ears, and only for BC-ABR compared to AC behavioural. BC-ABR threshold for $500 \mathrm{~Hz}$ was always better than $\mathrm{AC}$ behavioural thresholds (mean $=-16.7$; $\mathrm{SD}=10.8 ; t=$ $-3.8 ; d f=5, P=.013$ ). All difference scores were within $30 \mathrm{~dB}$.

Results of Study B indicate that BC-ABR accurately categorizes the degree of cochlear hearing loss at 2000 and $500 \mathrm{~Hz}$. Further, when $2000 \mathrm{~Hz}$ BC-ABR thresholds are elevated (i.e., $>30 \mathrm{dBnHL}$ at $2000 \mathrm{~Hz}$ ) and actual BC-ABR thresholds are obtained, the $\mathrm{BC}-\mathrm{ABR}$ thresholds are usually within $5 \mathrm{~dB}$ of the behavioural $\mathrm{BC}$ thresholds. Because few actual BC-ABR thresholds were obtained for $500 \mathrm{~Hz}$, conclusions are limited. 


\section{Discussion}

The present studies show that the $\mathrm{BC}$ tone $\mathrm{ABR}$ accurately differentiates normal versus elevated cochlear sensitivity in infants with conductive or sensorineural hearing loss, both for $500 \mathrm{~Hz}$ and $2000 \mathrm{~Hz}$ (Study A). Additionally, at least for $2000 \mathrm{~Hz}$, the degree of cochlear impairment can be accurately categorized (Study B). Finally, the present studies also determined difference scores which might be used to predict behavioural thresholds when elevated BC tone-ABR thresholds are obtained (Study B). These studies are the first to formally assess the performance (e.g., accuracy of categorization of hearing loss type, as well as degree of boneconduction elevation) of the $\mathrm{BC}$ tone-evoked $\mathrm{ABR}$ in groups of infants with conductive or sensorineural hearing loss. Prior to this, a long history of clinical use together with studies assessing infants with conductive hearing loss, and a few anecdotal or small-N studies with sensorineural hearing loss were relied on to support the use of $\mathrm{BC}$ tone $\mathrm{ABR}$ for clinical practice.

4.1. Study A. As shown in Study A, nearly all infants showing responses to $\mathrm{BC}$ tones at $30 \mathrm{dBnHL}$ at $2000 \mathrm{~Hz}$ (28/31 ears) and/or $20 \mathrm{dBnHL}$ at $500 \mathrm{~Hz}$ (36/37 ears) had normal cochlear sensitivity at these frequencies on followup. Similarly, all those infants with no response at these intensities had sensorineural hearing impairment $(2000 \mathrm{~Hz}$ : 107/107 ears; $500 \mathrm{~Hz}$ : 22/22 ears) on followup. Ears with mild sensorineural hearing loss (30-40 dBHL), for the most part (10/13 ears), showed elevated BC ABR. These results support the recommendations made by Stapells and colleagues to use these BC intensities to separate normal versus elevated cochlear sensitivity in infants $[10,11,15,16,29]$ and they are currently used by the BCEHP [4] and OIHP [8].

4.2. Study B. When bone-conduction responses are elevated (i.e., not present at the normal levels), it is useful to determine the degree of sensorineural elevation by assessing higher intensities. Until recently, the BCEHP protocol only required determination of "normal" versus "elevated" BC ABR, with threshold searching optional after completing other mandatory elements (the recently revised BCEHP protocol now requires determination of $\mathrm{BC}-\mathrm{ABR}$ thresholds at $2000 \mathrm{~Hz}$ (and at $500 \mathrm{~Hz}$ if the only AC elevation is at $500 \mathrm{~Hz}$ ) [4]). In practice, clinicians in the current study often tested the maximum $\mathrm{BC}$ level when no response was present at the normal level. In fewer cases, actual BC-ABR thresholds were obtained, typically at $2000 \mathrm{~Hz}$. Thus, for this study, for a reasonably large group of infants at $2000 \mathrm{~Hz}$ (29 ears with normal cochlear sensitivity; 75 ears with sensorineural hearing loss), we were able to determine that the $\mathrm{BC}$ ABR accurately estimated (92.8\% accuracy) cochlear status into three categories (i.e., normal; 30-65 dBHL; >65 dBHL). Similar accuracy was found for $500 \mathrm{~Hz}$ (94.7\%); however, interpretation for $500 \mathrm{~Hz}$ is limited due to the small number of ears with sensorineural hearing loss (37 ears with normal cochlear sensitivity; 10 ears with sensorineural hearing loss) that we were able to classify into the three categories at this frequency. The above results include both "certain" and "presumed" sensorineural hearing loss. Similar results were obtained when only "certain" cases were considered, although with about half the number of ears.

Ideally, one wants to know the correction factor that allows one to convert BC-ABR thresholds (in $\mathrm{dBnHL}$ ) to estimated behavioural BC thresholds (in $\mathrm{dBeHL}$ ). To do this, one needs actual BC-ABR thresholds. As noted previously, the earlier BCEHP protocol did not mandate BC-ABR thresholds, thus, actual thresholds were not obtained for most of the infants in this study. Additionally, actual BCABR thresholds were not available for the ears with normal cochlear sensitivity as testing was only carried out at the normal BC-ABR level. Further, many infants with significant sensorineural hearing loss had no response at the maximum level, and thus thresholds could not be determined. Therefore, when available, threshold data were only available for those infants whose hearing loss fell in the middle BCABR category. Actual threshold results for $2000 \mathrm{~Hz}$ were available for 21 ears that had both BC-ABR and $A C$ behavioural thresholds and 12 ears that had both BC-ABR and $\mathrm{BC}$ behavioural thresholds. The BC-ABR was, on average, essentially the same as the $\mathrm{BC}$ behavioural (BC ABR was $1.3 \mathrm{~dB}$ better than $\mathrm{BC}$ behavioural) and $6.2 \mathrm{~dB}$ better than the $\mathrm{AC}$ behavioural. Based on these results, a preliminary correction factor to estimate $\mathrm{BC}$ behavioural thresholds for $2000 \mathrm{~Hz}$ from the $\mathrm{BC} \mathrm{ABR}$ would be $0 \mathrm{~dB}$ (i.e., $40 \mathrm{dBnHL}$ $\mathrm{BC} \mathrm{ABR}=40 \mathrm{dBeHL} \mathrm{BC}$ behavioural); this is similar to published ABR-minus-behavioural difference scores for airconduction $\mathrm{ABR}$ at $2000 \mathrm{~Hz}[11,20]$. This is also consistent with little or no maturation of mid- to high-frequency BC thresholds, with infant thresholds within $5 \mathrm{~dB}$ of adult thresholds above $1000 \mathrm{~Hz}[20,30,38]$. The current data cannot provide a reliable correction factor for $500 \mathrm{~Hz} \mathrm{BC}$ $\mathrm{ABR}$. It is likely to be a larger correction (e.g., BC-ABR is $10-20 \mathrm{~dB}$ better than behavioural followup) as many studies have shown low-frequency BC thresholds to be significantly better in infants than in adults $[20,30,32,38]$.

4.3. Outliers. As noted in the results, in a few cases $(4 / 197$ ears), the BC-ABR categorization of normal versus elevated cochlear sensitivity differed from that of behavioural followup categorization. In one case, behavioural followup showed a moderate cochlear hearing loss, whereas the BC-ABR was normal and it is possible that the behavioural thresholds did not reflect true sensitivity (e.g., although behavioural results were considered to have "good" reliability, this child had a significant mental handicap). In the other three cases, behavioural followup showed mildly elevated thresholds compared to the normal $\mathrm{BC}$ ABR. There are several possible explanations. First, it is possible that the normal levels miss mild (30-40 dBHL) cochlear hearing loss. However, of the 13 ears with mild cochlear hearing loss in Study A, only 3 were missed by BC ABR. Equally likely, progressive and/ or late onset hearing loss can also explain these results as there were 5, 10, and 16 months between ABR and followup behavioural testing for these three children. Indeed, previous studies have shown that $5-30 \%$ of children with 
sensorineural hearing loss show progression in their thresholds (for review see: [39]), so it would be expected that a small number of children in our study would show worse thresholds on followup.

4.4. Limitations of This Study. As a retrospective chart-review study, it was not possible to ensure complete data were obtained for every child in the study (e.g., not every child had both frequencies/ears tested, and only a subset had actual BC-ABR thresholds). Threshold results for $500 \mathrm{~Hz}$ are particularly limited. Similarly, the timing and amount of data obtained at behavioural followup were variable. Without same day ABR and behavioural testing, changes in thresholds could not be controlled for.

4.5. Clinical Implications. The results of these studies have several key implications for clinical application. First, it is clear for both $2000 \mathrm{~Hz}$ and $500 \mathrm{~Hz}$ that normal/elevated cochlear sensitivity may be inferred from the presence/absence of a BC-ABR at $30 \mathrm{dBnHL}$ for $2000 \mathrm{~Hz}$ and $20 \mathrm{dBnHL}$ for $500 \mathrm{~Hz}$. This is consistent with previous recommendations and current diagnostic protocols.

When elevated, it is possible to categorize the degree of cochlear hearing loss, at least for $2000 \mathrm{~Hz}$. For elevated $2000 \mathrm{~Hz}$ results, if no BC-ABR is present at $60 \mathrm{dBnHL}$ then behavioural thresholds are greater than $65 \mathrm{dBHL}$. If $\mathrm{BC}-$ $\mathrm{ABR}$ is elevated but present between 35 and $60 \mathrm{dBnHL}$, then behavioural thresholds are 30-65 dBHL. If actual threshold is obtained, then the estimated BC behavioural threshold is equal to the $\mathrm{BC}-\mathrm{ABR}$ threshold (i.e., a correction factor of $0 \mathrm{~dB}$ ). This correction factor only applies when BC-ABR thresholds are elevated (i.e., does not apply if $\mathrm{BC} A B R$ is present at the normal level). For elevated $500 \mathrm{~Hz}$ results, Study B suggested that categorization is possible but limited ears with cochlear hearing loss preclude clinical application at this time.

Future studies should investigate BC-ABR and behavioural thresholds obtained on the same day in infants with a wide range of sensorineural hearing loss, especially mild hearing loss. Additional threshold results at $500 \mathrm{~Hz}$ are particularly needed. Finally, as there are very few data in the literature for 1000 and/or $4000 \mathrm{~Hz}$ BC ABR, future studies should also investigate these frequencies in children with normal and impaired hearing.

\section{References}

[1] C. Kennedy, D. McCann, M. J. Campbell, L. Kimm, and R. Thornton, "Universal newborn screening for permanent childhood hearing impairment: an 8-year follow-up of a controlled trial," Lancet, vol. 366, no. 9486, pp. 660-662, 2005.

[2] C. Yoshinaga-Itano and J. S. Gravel, "The evidence for universal newborn hearing screening," American Journal of Audiology, vol. 10, no. 2, pp. 62-64, 2001.

[3] C. Yoshinaga-Itano, A. L. Sedey, D. K. Coulter, and A. L. Mehl, "Language of early- and later-identified children with hearing loss," Pediatrics, vol. 102, no. 5, pp. 1161-1171, 1998.
[4] British Columbia Early Hearing Program (BCEHP), BCEHP Audiology Assessment Protocol, 2012, http://www.phsa.ca/ AgenciesAndServices/Services/BCEarlyHearing/ForProfessionals/Resources/Protocols-Standards.htm.

[5] J. Busa, J. Harrison, J. Chappell et al., "Year 2007 position statement: principles and guidelines for early hearing detection and intervention programs," Pediatrics, vol. 120, no. 4, pp. 898921, 2007.

[6] American Speech-Language-Hearing Association (ASHA), Guidelines for the audiologic assessment of children from birth to 5 years of age, http://www.asha.org/policy/GL2004-00002.htm.

[7] Canadian Working Group on Childhood Hearing, Early Hearing and Communication Development: Canadian Working Group on Childhood Hearing (CWGCH) Resource Document, 2005, http://www.phac-aspc.gc.ca/publicat/eh-dp/index-eng .php.

[8] Ontario Infant Hearing Program (OIHP), 2008 Audiologic Assessment Protocol, 2009, http://www.mountsinai.on.ca/care/ infant-hearing-program/resolveUid/b84c6bf5acbf70c86347 eecd6be66b1f.

[9] Y. S. Sininger and M. L. Hyde, "Auditory brainstem response in audiometric threshold prediction," in Handbook of Clinical Audiology, J. Katz, L. Medwetsky, R. Burkard, and L. Hood, Eds., pp. 293-321, Lippingcott, Williams \& Wilkins, Baltimore, Md, USA, 6th edition, 2009.

[10] D. R. Stapells, "Frequency-specific evoked potential audiometry in infants," in A Sound Foundation Through Early Amplification, R. C. Seewald, Ed., pp. 13-31, Phonak AG, Stäfa, Switzerland, 2000.

[11] D. R. Stapells, "Frequency-specific threshold assessment in young infants using the transient ABR and the brainstem ASSR," in Comprehensive Handbook of Pediatric Audiology, R. C. Seewald and A. M. Tharpe, Eds., pp. 409-448, Plural, San Diego, Calif, USA, 2011.

[12] J. J. Eggermont, "The inadequacy of click-evoked auditory brainstem responses in audiological applications," Annals of the New York Academy of Sciences, vol. 388, pp. 707-709, 1982.

[13] T. W. Picton, "The strategy of evoked potential audiometry," in Early Diagnosis of Hearing Loss, S. E. Gerber and G. T. Mencher, Eds., pp. 297-307, Grune \& Stratton, New York, NY, USA, 1978.

[14] T. W. Picton and D. R. Stapells, “A "Frank's Run” latencyintensity function," in The Auditory Brainstem Response, J. T. Jacobson, Ed., pp. 410-413, College-Hill Press, San Diego, Calif, USA, 1985.

[15] D. R. Stapells and P. Oates, "Estimation of the pore-tone audiogram by the auditory brainstem response: a review," Audiology and Neuro-Otology, vol. 2, no. 5, pp. 257-280, 1997.

[16] D. R. Stapells, "Auditory brainstem response assessment of infants and children," Seminars in Hearing, vol. 10, no. 3, pp. 229-251, 1989.

[17] R. K. Karzon and J. E. Cho Lieu, "Initial audiologic assessment of infants referred from well baby, special care, and neonatal intensive care unit nurseries," American Journal of Audiology, vol. 15, no. 1, pp. 14-24, 2006.

[18] S. Windmill and I. M. Windmill, "The status of diagnostic testing following referral from universal newborn hearing screening," Journal of the American Academy of Audiology, vol. 17, no. 5, pp. 367-378, 2006.

[19] D. R. Stapells, J. S. Gravel, and B. A. Martin, "Thresholds for auditory brain stem responses to tones in notched noise from infants and young children with normal hearing or sensorineural hearing loss," Ear and Hearing, vol. 16, no. 4, pp. 361371, 1995. 
[20] K. R. Vander Werff, B. A. Prieve, and L. M. Georgantas, "Infant air and bone conduction tone burst auditory brain stem responses for classification of hearing loss and the relationship to behavioral thresholds," Ear and Hearing, vol. 30, pp. 350$368,2009$.

[21] G. R. I. Rodrigues and D. R. Lewis, "Threshold prediction in children with sensorioneural hearing loss using the auditory steady-state responses and tone-evoked auditory brain stem response," International Journal of Pediatric Otorhinolaryngology, vol. 74, no. 5, pp. 540-546, 2010.

[22] C. Y. Lee, T. H. Hsieh, S. L. Pan, and C. J. Hsu, "Thresholds of tone burst auditory brainstem responses for infants and young children with normal hearing in Taiwan," Journal of the Formosan Medical Association, vol. 106, no. 10, pp. 847-853, 2007.

[23] C. Y. Lee, F. S. Jaw, S. L. Pan, T. H. Hsieh, and C. J. Hsu, "Effects of age and degree of hearing loss on the agreement and correlation between sound field audiometric thresholds and tone burst auditory brainstem response thresholds in infants and young children," Journal of the Formosan Medical Association, vol. 107, no. 11, pp. 869-875, 2008.

[24] G. Rance, D. Tomlin, and F. W. Rickards, "Comparison of auditory steady-state responses and tone-burst auditory brainstem responses in normal babies," Ear and Hearing, vol. 27, no. 6, pp. 751-762, 2006.

[25] F. M. Ribeiro and R. M. Carvallo, "Tone-evoked ABR in fullterm and preterm neonates with normal hearing," International Journal of Audiology, vol. 47, no. 1, pp. 21-29, 2008.

[26] J. S. Gravel, "Potential pitfalls in the audiological assessment of infants and young children," in A Sound Foundation Through Early Amplification 2001, R. C. Seewald and J. S. Gravel, Eds., Proceedings of the 2nd International Conference, pp. 85-101, Phonak AG, Stäfa, Switzerland, 2002.

[27] J. S. Gravel, D. Kurtzberg, D. R. Stapells, H. G. Vaughan, and I. F. Wallace, "Case studies," Seminars in Hearing, vol. 10, no. 3, pp. 272-287, 1989.

[28] E. H. J. F. Boezeman, T. S. Kapteyn, L. Feenstra, and A. M. Snel, "Verification of the air-bone gap using cancellation and evoked responses," Audiology, vol. 24, no. 3, pp. 174-185, 1985.

[29] D. R. Stapells and R. J. Ruben, "Auditory brain stem responses to bone-conducted tones in infants," Annals of Otology, Rhinology and Laryngology, vol. 98, no. 12 I, pp. 941-949, 1989.

[30] J. J. Foxe and D. R. Stapells, "Normal infant and adult auditory brainstem responses to bone-conducted tones," Audiology, vol. 32, no. 2, pp. 95-109, 1993.

[31] B. Cone-Wesson, "Bone-conduction ABR tests," American Journal of Audiology, vol. 4, pp. 14-19, 1995.

[32] B. Cone-Wesson and G. M. Ramirez, "Hearing sensitivity in newborns estimated from ABRs to bone-conducted sounds," Journal of the American Academy of Audiology, vol. 8, no. 5, pp. 299-307, 1997.

[33] J. M. K. Nousak and D. R. Stapells, "Frequency specificity of the auditory brain stem response to bone- conducted tones in infants and adults," Ear and Hearing, vol. 13, no. 2, pp. 87-95, 1992.

[34] D. R. Stapells, “Threshold estimation by the tone-evoked auditory brainstem response: a literature meta-analysis," Journal of Speech-Language Pathology and Audiology, vol. 24, pp. 74-83, 2000.

[35] P. E. Campbell, C. M. Harris, S. Hendricks, and T. Sirimanna, "Bone conduction auditory brainstem responses in infants," Journal of Laryngology and Otology, vol. 118, no. 2, pp. 117122, 2004.
[36] American Academy of Pediatrics, "American Academy of Pediatrics Committee on Drugs: guidelines for monitoring and management of pediatric patients during and after sedation for diagnostic and therapeutic procedures," Pediatrics, vol. 89, no. 6, pp. 1110-1115, 1992.

[37] K. L. Napoli, C. G. Ingall, and G. R. Martin, "Safety and efficacy of chloral hydrate sedation in children undergoing echocardiography," Journal of Pediatrics, vol. 129, no. 2, pp. 287291, 1996.

[38] S. A. Small and D. R. Stapells, "Maturation of bone conduction multiple auditory steady-state responses," International Journal of Audiology, vol. 47, no. 8, pp. 476-488, 2008.

[39] S. Berrettini, F. Ravecca, S. Sellari-Franceschini, F. Matteucci, G. Siciliano, and F. Ursino, "Progressive sensorineural hearing loss in childhood," Pediatric Neurology, vol. 20, no. 2, pp. 130136, 1999. 


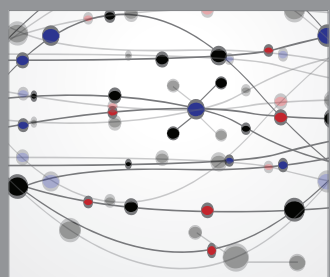

The Scientific World Journal
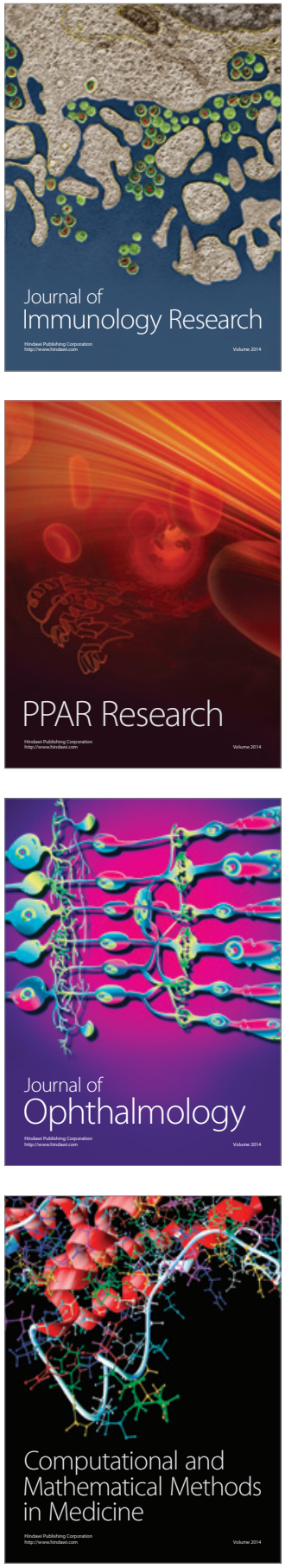

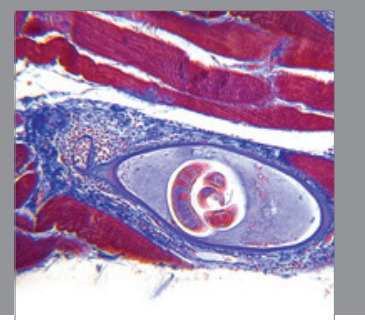

Gastroenterology

Research and Practice
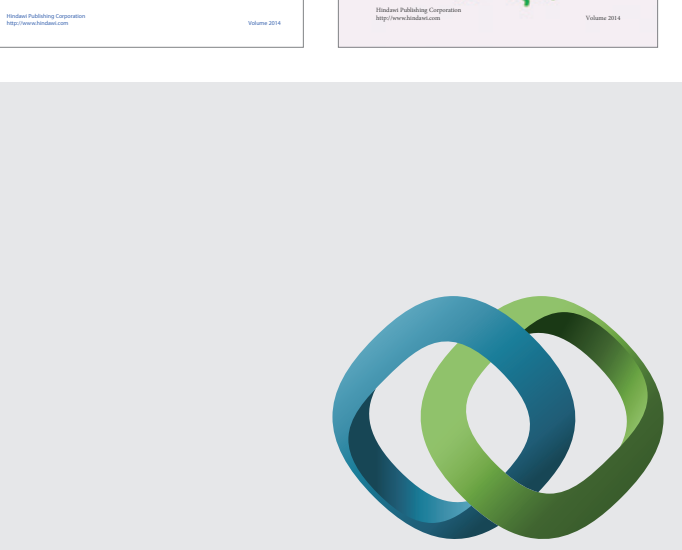

\section{Hindawi}

Submit your manuscripts at

http://www.hindawi.com
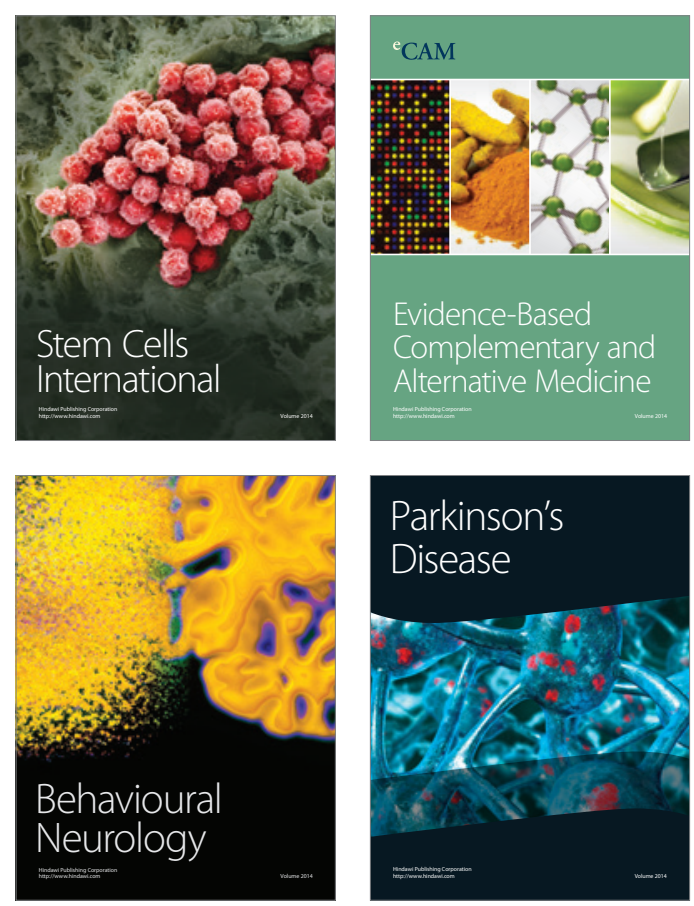

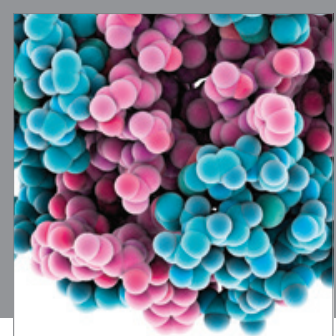

Journal of
Diabetes Research

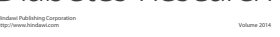

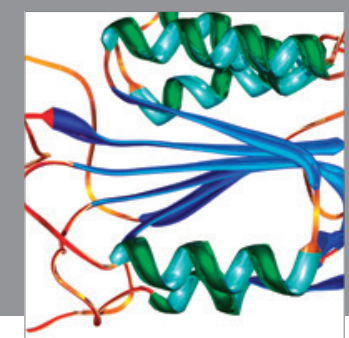

Disease Markers
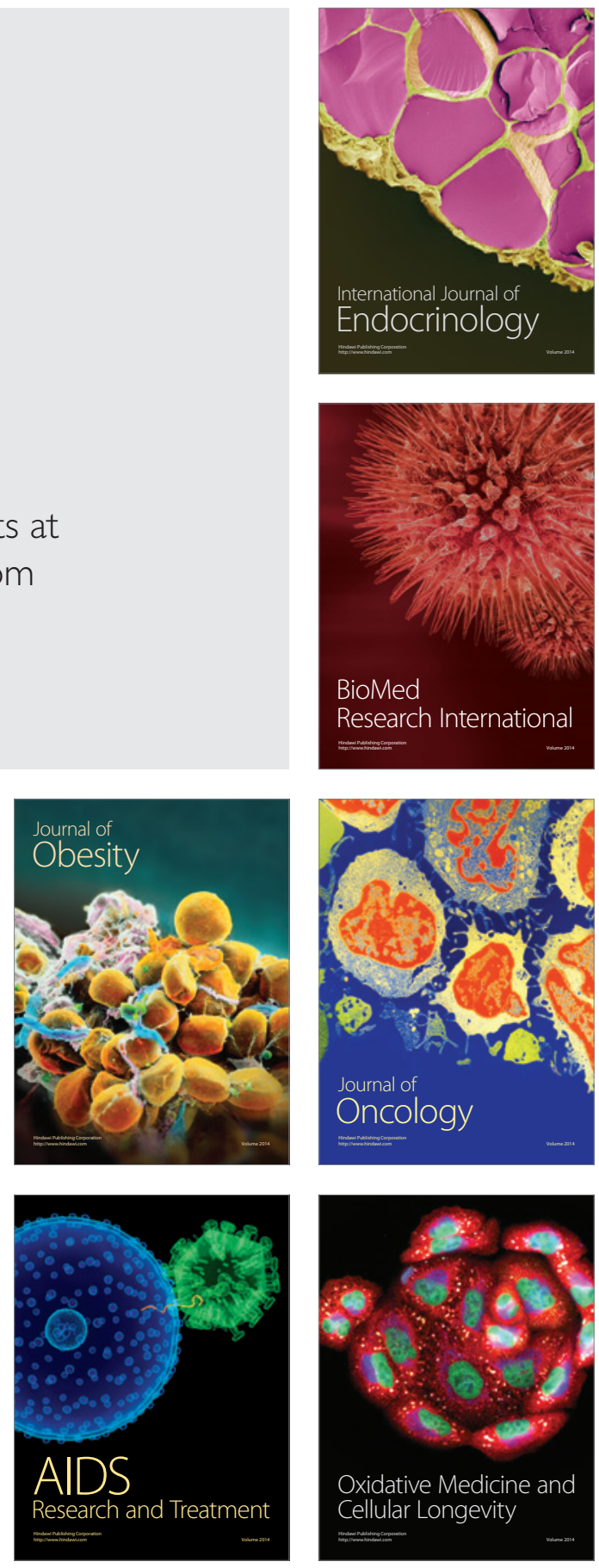